\title{
Direct identification of key intermediates in containers using Fourier-transform near-infrared spectroscopy through the protective polyethylene primary packaging
}

\author{
M. Ulmschneider ${ }^{1, *}$ and E. Pénigault ${ }^{2}$ \\ ${ }^{1}$ Pharmaceutical Quality Control and Assurance F. Hoffmann - La Roche Ltd, CH-4070 Basle, Switzerland \\ ${ }^{2}$ Laboratoire de Photochimie Générale, UMR 7525 du CNRS,ENSCMu, 3 rue Alfred Werner, \\ 68093 Mulhouse Cedex, France
}

\begin{abstract}
A fast and non-destructive Fourier-transform near-infrared (NIR) spectroscopy method with corresponding chemometric calibrations was developed for the identification of key intermediates of pharmaceutical active substances through the protective polyethylene (PE) primary packaging in containers. The samples were measured with a laboratory spectrometer. NIR spectra of incoming containers samples were also recorded for calibration with a spectrometer installed in the warehouse. The spectra of the different samples were recorded by diffuse reflectance with a $2 \mathrm{~m}$ long glass fiber optics probe in direct contact with the PE-protected surface of the material. The NIR spectra of the same products were stored in files and principal component analysis combined with cluster analysis were used to refine the identification criteria. To achieve validation of the resulting mathematical models, the samples were split into a calibration set and a validation set. The latter was used for external and independent tests. The resulting NIR application can be transferred to any spectrometer of the same type.
\end{abstract}

Keywords. Near-IR spectroscopy - key intermediates - pharmaceutical active substances - noninvasive identification - primary packaging - polyethylene.

\section{Introduction}

Identification by NIR spectroscopy of pharmaceutical raw materials like active ingredients or excipients is now a well established technique $[1,2,3,4]$. To comply with current regulatory requirements $[5,6]$ many pharmaceutical companies perform a containerwise identity testing of all incoming materials, either in the warehouse, at the production line, or in the quality control laboratory. Nowadays, a fast and nondestructive method like NIR spectroscopy implemented in the warehouse is proposed as an alternative to other analytic methods in the laboratory, when large numbers of identity tests are carried out $[7,8]$. This avoids the collection and disposal of samples as well as a laboratory infrastructure and a traceability system which links the sample to the corresponding container. To overcome strong restricting working conditions or possible cross-contaminations when active or dangerous substances are manipulated in the warehouse, the authors set out to identify the key intermediates directly in the opened containers by keeping the protective polyethylene bag sealed. This can be achieved by combining a Fourier-transform NIR spectrometer with an optical fibre probe and adequate multivariate calibrations. This technique was recommended at the Pharmaceutical Division of F. Hoffmann-La Roche Ltd in Basle to identify selected key intermediates of pharmaceutical active substances coming into the warehouse. The NIR model calculation and calibration were performed in the laboratory. Once validated, the NIR application was transferred to the spectrometer installed in the warehouse.

\section{Experimental}

The NIR range (1000 to $2500 \mathrm{~nm}$ ) covers overtones and combination vibrations occurring in the mid-infrared range of the $-\mathrm{OH},-\mathrm{NH},-\mathrm{CH}$, and $-\mathrm{SH}$ groups. As the intensity bands in the NIR region is 10 to 100 times weaker as that of bands in the mid-IR region, dilution of the samples is not necessary when spectra are recorded $[9,10]$. The use of a greater volume considerably reduces handling and preparation time of the samples. Since glass is not an obstacle to NIR radiation, the use of fibre optic probes makes it possible to bring NIR light from the spectrometer directly to a remote sample and, then, to collect the spectrum measured by diffuse reflection. Two Fourier-Transform Near-Infrared birefringent interferometers of the same type (NIRVIS, supplied by Buhler Anatec, Uzwil, Switzerland), were used for the present study. One spectrometer (serial $\mathrm{N}^{\circ}$ 95/1197) was located in the laboratory and the second one (serial $\mathrm{N}^{\circ} 1378$ ) was operated in the warehouse. The measurements were performed by using a standard $2 \mathrm{~m}$ stellar-patterned bi-directional glass fibre optics bundle for reflectance measurements, supplied by Buhler Anatec and connected to the 
spectrometer. Repeatability of instrument performance was ensured through a daily suitability test and by measuring a reference spectrum at regular intervals and when going on to a new substance. The programme used for communication with the spectrometer, data acquisition, and calibration, was the BCAP 6.00 software, supplied by Buhler Anatec. All the samples or spectra used in the present work were collected from containers of regular production batches which were in compliance with the specifications of the corresponding product. Samples of one active substance, Fleroxacin, four key intermediates, ACT, MAEM (two key intermediates of Rocephin ${ }^{\circledR}$ ), Ethoxyfluorouracil (a key intermediate of Fleroxacin), and PTH-Aminoalcohol (a key intermediate of Saquinavir) were collected and transferred to the laboratory for NIR spectroscopy. The substances were initially delivered to the warehouse in sealed PE bags, which in turn were contained in drums or box containers. To simulate the protective primary PE-packaging, the samples were filled into small PE-bags. The polyethylene material specific to each supplier was used. The NIR-spectra of two additional key intermediates, HPD-Chloralcohol (a key intermediate of Nelfinavir) and Orlistat (unmilled material) were recorded directly in the warehouse in the original containers through the primary packaging without sampling. The substances were inspected by bringing the fibre optics in contact with the active material through the protective PE foil. The operator had to control the absence of air bubbles between the foil and the window of the fibre probe. Each sample was measured three times (in the laboratory) or twice (in the warehouse). The NIR spectra were recorded with one scan at a resolution of $25 \mathrm{~cm}^{-1}$, moving the probe and varying the pressure on the sample between two measurements. The number of sampled containers depended from the availability of the material. The number of spectra recorded per substance and batch is given in table I. For MAEM more batches were available as for ACT or Fleroxacin. For each batch of key-intermediate tested in the laboratory only one sample could be prepared. On the other hand, up to five containers per batch could be tested in the warehouse. Example spectra of the six key intermediates and active substance recorded through PE foils are shown in figure 1.

\section{Calibration and validation}

The NIR spectra of the samples were collected to form a database which was split into a learning set and a training set. Table I gives the number of spectra of each sampled batch of key intermediate in the learning and the training set. The NIR model was calculated and validated by using the principal component analysis (PCA) and cluster calibration modules of BCAP. During calibration, spectra of analogous substances were grouped in properties and the different spectra had to be separated simultaneously. The NIR spectra in the learning set were compressed via principal component analysis, i.e. the spectral data was deconvoluted into corresponding primary factors by a suitable linear coordinate transformation in the spectral space $[11,12]$. The key information which describes systematic variations between
Table I. List of the six pharmaceutical key intermediates and active substance to be identified by NIR-spectroscopy, with number of spectra and samples stored in learning and training sets.

\begin{tabular}{|c|c|c|c|}
\hline \multirow[t]{2}{*}{ Substances } & \multirow[t]{2}{*}{$\begin{array}{c}\text { Batch } \\
N^{\circ}\end{array}$} & \multicolumn{2}{|c|}{$\begin{array}{c}\text { Number of } \\
\text { spectra (samples) in }\end{array}$} \\
\hline & & $\begin{array}{c}\text { learning } \\
\text { set }\end{array}$ & $\begin{array}{c}\text { training } \\
\text { set }\end{array}$ \\
\hline \multirow{5}{*}{ ACT } & 1 & $3(1)$ & \\
\hline & 2 & $3(1)$ & \\
\hline & 3 & $3(1)$ & \\
\hline & 4 & & $3(1)$ \\
\hline & 5 & & $3(1)$ \\
\hline \multirow{9}{*}{ MAEM } & 1 & $3(1)$ & \\
\hline & 2 & $3(1)$ & \\
\hline & 3 & $3(1)$ & \\
\hline & 4 & $3(1)$ & \\
\hline & 5 & $3(1)$ & \\
\hline & 6 & $3(1)$ & \\
\hline & 7 & & $3(1)$ \\
\hline & 8 & & $3(1)$ \\
\hline & 9 & & $3(1)$ \\
\hline \multirow{5}{*}{$\begin{array}{l}\text { Ethoxyfluoro- } \\
\text { uracil }\end{array}$} & 1 & $3(1)$ & \\
\hline & 2 & $3(1)$ & \\
\hline & 3 & $3(1)$ & \\
\hline & 4 & & $3(1)$ \\
\hline & 5 & & $3(1)$ \\
\hline \multirow{5}{*}{$\begin{array}{l}\text { PTH- } \\
\text { Aminoalcohol }\end{array}$} & 1 & $3(1)$ & \\
\hline & 2 & $3(1)$ & \\
\hline & 3 & $3(1)$ & \\
\hline & 4 & & $3(1)$ \\
\hline & 5 & & $3(1)$ \\
\hline \multirow{3}{*}{ Fleroxacin } & 1 & $15(5)$ & \\
\hline & 2 & $15(5)$ & \\
\hline & 3 & & $15(5)$ \\
\hline \multirow{5}{*}{$\begin{array}{l}\text { HPD- } \\
\text { Chloralcohol }\end{array}$} & 1 & $10(5)$ & \\
\hline & 2 & $10(5)$ & \\
\hline & 3 & $10(5)$ & \\
\hline & 4 & & $10(5)$ \\
\hline & 5 & & $6(3)$ \\
\hline \multirow{5}{*}{ Orlistat } & 1 & $10(5)$ & \\
\hline & 2 & $10(5)$ & \\
\hline & 3 & 7 (3) & \\
\hline & 4 & & $10(5)$ \\
\hline & 5 & & $10(5)$ \\
\hline
\end{tabular}

groups of substance spectra was contained in the first few primary factors. These formed a linearly independent orthogonal axis system in which each spectrum was represented by a point. The effectiveness of the spectral deconvolution was assessed during the calibration step by calculating residual values which determined the boundaries of the model as a noise threshold value. Cluster separation algorithms in 


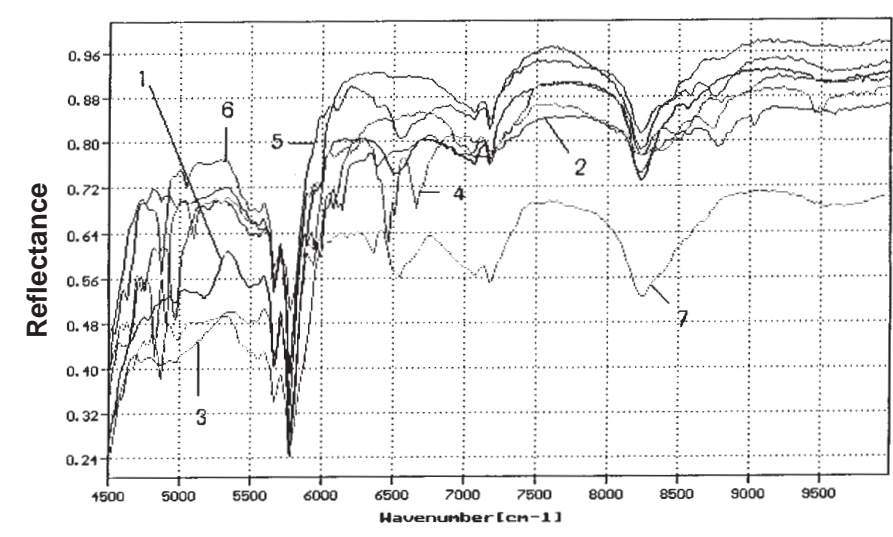

Figure 1. Raw NIR spectra of the six pharmaceutical key intermediates and active substance recorded through polyethylene: ACT (1), MAEM(2), Ethoxyfluorouracil (3), PTH-Aminoalcohol (4), Fleroxacin (5), HPD-Chloralcohol (6), Orlistat (7).

BCAP are based on the soft modeling approach which is also implemented in an other commercially available software package [13]. It was suggested that a higher selectivity can be achieved when those primary factors are selected which correlate best with the properties. The secondary factors focus on the separation of the properties and are not necessarily the factors which account for the greatest variance during the PCA. For each calibration spectrum a tolerance radius was then determined by using that smaller set of secondary factors. This radius defined a hypersphere centered on the corresponding spectrum in the factor space. For a given substance all spectra i.e. hyperspheres cluster into a "cloud". The spectrum of any unknown sample was positioned into the factor space by its coordinates (loadings). Identification of the unknown substance was confirmed if the spectrum lay within the corresponding cluster and if simultaneously the residual value was within the boundaries of the model.

The cluster model for identity testing was computed by using the 132 original spectra in the learning set. The best calibration was obtained by using normalization and first derivative, the spectral ranges from 4500 to $5496 \mathrm{~cm}^{-1}, 6012$ to $8076 \mathrm{~cm}^{-1}$, and 8508 to $9120 \mathrm{~cm}^{-1}$, four primary factors and two secondary factors (factors $\mathrm{N}^{\circ} 3$ and 4 were selected for radius determination and property separation). The calculated maximum allowed residual was 0.02172 . Some idea of the selectivity of the cluster model can be seen from the factor plot of figure 2, which is the projection of the factor space on the hyperplane defined by factors $\mathrm{N}^{\circ} 3$ and 4 . Each spectrum is represented as an ellipsis and corresponds to the radial extension of the spectral hypersphere in the factor space. Spectra of similar substances are grouped together in clusters. The boundaries of the groups are defined by the hyperspheres around the spectra of each sample. As revealed by the figure, the different clusters do not overlap i.e. are separate.

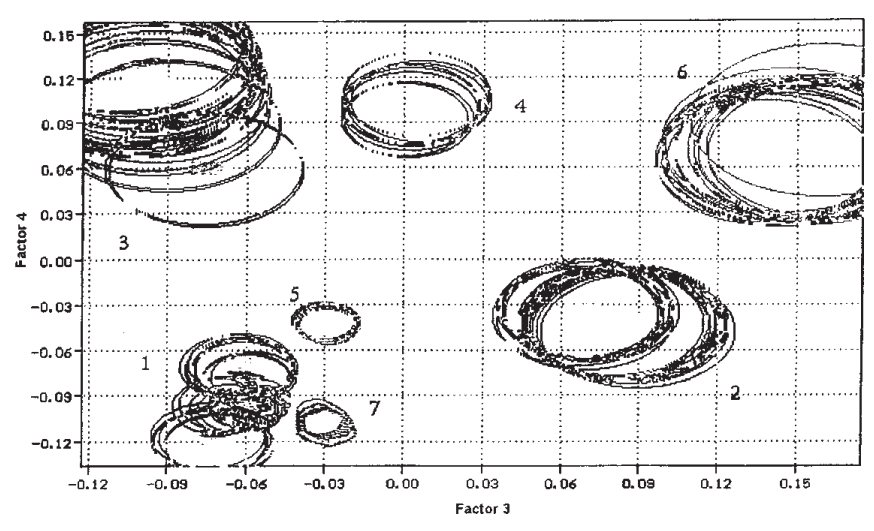

Figure 2. Projection of spectral space on the hyperplane defined by factors No 3 and No 4. Displayed are the 132 spectra in the series: ACT (1), MAEM (2), Ethoxyfluorouracil (3), PTH-Aminoalcohol (4), Fleroxacin (5), HPD-Chloralcohol (6), Orlistat (7).

In addition to the mapping of the spectra of the six key intermediates and active substance into cluster regions in the spectral space, the following validation steps determined the ability of the NIR models to predict the identity of unknown samples correctly. The BCAP 6.00 software proposes an internal cross-validation procedure. Each model (library or cluster) can be tested against all others. BCAP will scan each spectrum in each model and predict identity with the currently tested model. As long as the present study was going on, 6 models for the identification of 17 active substances from the regular production of different Roche plants and the corresponding spectral files (with more than 500 spectra) were available on the same system. No interference was found between already existing models. An external validation of the models was also performed with the independent training set. This is the safest way to test the NIR model for routine application. An off-line prediction of identity testing was performed with the training set. The score for correct identification reached $100 \%$ for the complete training set.

A calibration transfer was performed from the laboratory spectrometer to the equipment located in the warehouse. Copies of the spectral files corresponding to the learning and training sets of the active substance and the key intermediates were realized on the computer of the NIRVIS spectrometer located in the warehouse. NIR models identical to those obtained in the laboratory were calculated. In addition to the validation of the calibration with the independent training set, containers of the selected substances were tested for identity to qualify the performance of the complete system.

\section{Discussion}

The method is fast (a few seconds), non-destructive and can easily be performed directly through the sealed protective 


\section{Original articles}

PE-bag in the original containers. Sample preparation is not required apart from the opening and closing of the drum or box containers. This is particularly important for a non invasive identity testing. Cross contaminations are avoided and no cleaning of the tip of the light probe is required. The operators can work without special protective clothing or equipment. Genuine experience with identity testing through protective packaging or polyethylene foils was first gained at Roche in the early 90s with NIR applications aimed at identifying solid excipients. Although the method proved feasible and effective, the large number (up to 100) of excipients that must be identified and the occurrence of numerous similarities in structure or quality of the substances resulted into a time consuming maintenance of the application, which was not considered as reliable enough for proper performance in a warehouse. For active ingredients and raw materials the question of measuring through the protective packaging was reconsidered. The selectivity of the NIR spectroscopy is due to the measurement of structural and physical properties, combined with the corresponding chemometrical calibrations. The spectra of the six pharmaceutical key intermediates and active substance also contained information on the way the measurement was performed through the PE bag. Although PE bags are thin and transparent, the NIR spectrum measured through it will include more or less sharp additional bands. Polyethylene bands occur at 8250,7250 , and $5800 \mathrm{~cm}^{-1}$, but the overall NIR band structure of the raw materials is maintained. This is shown in figure 3. The raw NIR spectrum of one sample of Fleroxacin is compared to the spectrum of a sample measured through 1 , respectively 2 polyethylene foils. The PE bands do not alter the overall shape of the NIR spectrum of Fleroxacin. The spectra of two other active substances not included in the present study, lidocaine hydrochloride and pyrimethamine, are also given and show the same features. Accordingly, the presence of the PE bands is not expected to alter the selectivity of the NIR model. Mathematical pretreatments compensate for the differences in spectrum intensity and baseline shifts.

\section{Conclusion}

An application of NIR spectroscopy was developed and validated by using spectra recorded with a NIRVIS spectrometer installed in the laboratory and from a NIRVIS spectrometer located in the warehouse. Model calculation and validation were conducted in the laboratory. Once validated, the NIR database was simply transferred to the equipment available in the warehouse and the NIR model again calculated. Direct transfer of NIR models from one instrument to another is usually not straightforward $[14,15]$. However, a reliable transfer of complete spectral data sets from one NIRVIS to another was achieved. This permitted to avoid systematic repetition of measurements for the same sample on both spectrometers and considerably reduced the time required for implementation of the NIR application. It also allowed a more flexible building up of spectral libraries with spectra originating from different equipments and locations.
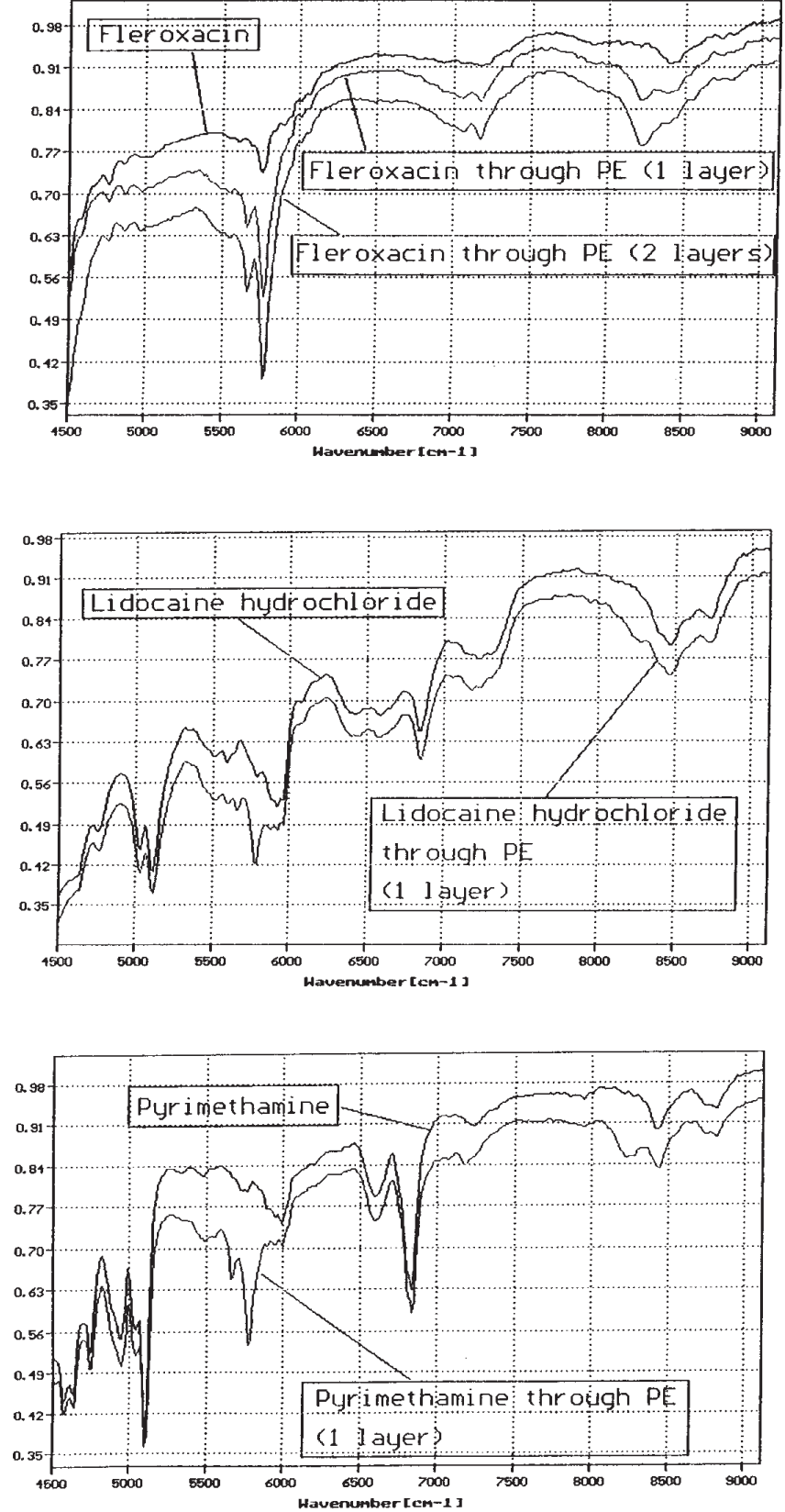

Figure 3. NIR spectra of Fleroxacin, lidocaine hydrochloride, and pyrimethamine recorded with and without polyethylene foils.

\section{References}

1. Hailey, P.A. European Pharmaceutical review 1996, 6, 45-49.

2. Brutsche, A. European Pharmaceutical review 1996, 9, 45-51.

3. Plugge, W.; van der Vlies, C. J. of Pharm. \& Biomed. Anal. 1992, 10, 787-803.

4. Ulmschneider, M.; Wunenburger, A.; Pénigault, E. Analusis 2000, to be published. 


\section{Original articles}

5. Ergänzende Leitlinie für die Probenahme von Ausgangsstoffen, C.6.2, 7. Lfg., 1993, Oeser/Sander, PharmBetrV-Kommentar.

6. EC GMP-Guide, Guide to good manufacturing practice, 5.30, "Starting Materials", 1992.

7. Gemperline, P.J.; Webber, L.D.; Cox F.O. Anal. Chem. 1989, $61,138-144$

8. Kohn, W.H.; Jeger, A.N. Journal of Forensic Sciences 1992, 37, 34-41.

9. Bokobza, L.; J. Near Infrared Spectroscopy 1998, 6, 35-41.

10. Bonano, A.S.; Olinger, J.M.; Griffiths, P.R. in Near-Infrared Spectroscopy; Hildron, K.I.; Isaksson, T.; Naes, T.; Tandberg, A. Eds., Ellis Horwood Ltd: London, 1992, Chapter 3.
11. Wold, S.; Esbensen, K.; Geladi, P. Chemometrics and Intelligent Laboratory Systems 1987, 2, 37-52.

12. Massart, D.L.; Vandeginste, B.G.M.; Deming, S.N.; Michotte, Y.; Kaufman, L. Eds., Chemometrics: a textbook; Elsevier: Amsterdam, 1988, Chapter 21.

13. Griffin, J.; Grummisch, U. in Near-Infrared Spectroscopy; Hildron, K.I.; Isaksson, T.; Naes, T.; Tandberg, A. Eds., Ellis Horwood Ltd: London, 1992, Chapter 13.

14. Hoffmann, U.; Zanier-Szydlowski, N. J. Near Infrared Spectroscopy 1999, 7, 11-45.

15. Bouveresse, E.; Massart, D.L.; Dardenne, P. Analytica Chimica Acta 1997, 297, 405-416. 